\title{
Comprensión de la lectura de textos instruccionales para la práctica de juegos deportivos. Estrategias cognitivas y metacognitivas aplicadas por los estudiantes
}

\author{
Understanding the reading of instructional texts for the practice of sports \\ games. Cognitive and metacognitive strategies applied by students
}

\author{
- GLENIA VÁSQUEZ \\ gleniavasquez@gmail.com \\ Código ORCID: 0000-0002-8279-9113 \\ Unidad Educativa Municipal Cecilio Acosta, Venezuela
}

Artículo recibido en abril 2019 / Arbitrado en mayo 2019 / Publicado en julio 2019

\begin{abstract}
Resumen El presente trabajo de investigación tuvo como objetivo identificar cuáles de las estrategias cognitivas y metacognitivas emplean los estudiantes de Educación Básica para la comprensión de los textos instruccionales utilizados para la práctica de los juegos deportivos en el área de Educación Física. La metodología se basó en un diseño cuantitativo, bajo la modalidad de investigación de campo, de carácter descriptivo. Los participantes fueron diecinueve estudiantes de $5^{\circ}$ grado de la Unidad Educativa Estadal Bolivariana "Ricardo Zuloaga" a los que se les aplicó el test ACRA (Román y Gallegos, 1994). Los resultados evidenciaron que las estrategias cognitivas fueron empleadas "siempre", específicamente, las estrategias de "codificación de la información"; mientras que las estrategias metacognitivas fueron "algunas veces", con respecto a sus procesos de planificación y autorregulación. Estos resultados permitieron demostrar a los docentes lo beneficioso que resulta para los estudiantes adquirir y poner en práctica dichas estrategias.
\end{abstract}

Palabras clave: Estrategias cognitivas; estrategias metacognitivas; textos instruccionales; juegos deportivos; Educación Física

\begin{abstract}
This research work aimed to identify which of the cognitive and metacognitive strategies used by Basic Education students to understand the instructional texts used to practice sports games in the area of Physical Education. The methodology was based on a quantitative design, under the field research modality, of a descriptive nature. The participants were nineteen 5th grade students of the Bolivarian State Educational Unit "Ricardo Zuloaga" to whom the ACRA test was applied (Román and Gallegos, 1994). The results showed that cognitive strategies were used "always", specifically, the "information coding" strategies; while the metacognitive strategies were "sometimes", regarding their planning and selfregulation processes. These results allowed teachers to demonstrate how beneficial it is for students to acquire and implement these strategies.
\end{abstract}

Keywords: Cognitive strategies; metacognitive strategies; instructional texts; sports games; Physical Education 


\section{INTRODUCCIÓN}

Los quehaceres del día a día se encuentran inmersos en un mundo de cosas que giran a nuestro alrededor y que sin darnos cuenta, en muchas ocasiones, logramos obtener cierto aprendizaje de ello. No es sino después de un tiempo que lo identificamos cuando se nos presenta alguna situación parecida a vivencias anteriores.

Vale resaltar que de esas vivencias obtenemos las habilidades para emplear soluciones a problemas que se nos plantean; esas destrezas constituyen un procedimiento estructurado que puede ser amplio $y$ adaptable, y que a su vez también se renueva ante distintas situaciones. Este proceso es denominado estrategia.

La palabra estrategia, en sus inicios, fue empleada con fines de maniobras para contrarrestar ataques de guerra, y así crear acciones con fines de evitar o vencer algún combate, dando así prioridad al empleo de la inteligencia.

En este sentido, Weihrich y Koontz (1993) plantean que

(...) el término estrategia se deriva de la palabra griega strategos y significa: general. Este término fue usado durante años por el ejército con el fin de aludir a los grandes planes o ventajas que el enemigo no poseía y que se mantenían en secreto para ganar las batallas ( $p$. 123).

Según lo planteado por los autores anteriormente mencionados, el término estrategia hacía referencia a la creación y empleo de grandes planes que se mantenían en secreto para aventajar al enemigo durante las batallas y así obtener la victoria.

Actualmente es empleado tanto para hacer mención a habilidades militares, como además empresariales, para la promoción de productos o comercios. Adicionalmente ha evolucionado en el plano educativo para el desarrollo y avance del sistema pedagógico.

En referencia al sistema educativo, las estrategias están enfatizadas en la toma de decisiones y acciones de forma consciente, con el fin de proporcionar, por parte del docente, y obtener del estudiante, un aprendizaje significativo; de allí que entre ellas se mencione a las estrategias de aprendizaje que emplean los estudiantes (estrategias cognitivas y estrategias metacognitivas) para el logro de ese aprendizaje significativo.

Sobre la base de lo planteado anteriormente, vale acotar que lo cognitivo hace mención al proceso que utiliza la persona para adquirir el conocimiento; por ello habría que indicar que las estrategias cognitivas las aplica el estudiante (en este caso) para que lo ayuden a comprender.

Respecto a ello, González y Tourón (1992) plantean que son "un conjunto de estrategias que se utilizan para aprender, codificar, comprender y recordar la información al servicio de unas determinadas metas de aprendizaje" (p. 57).

Basado en lo que plantean estos autores, se trata de una serie de acciones conscientes que permiten a quienes las utilicen instruirse, recopilar, alcanzar y memorizar información que les proporcionan el alcance de objetivos para el logro de un aprendizaje. 
Por otro lado, la metacognición representa el proceso consciente de regulación de las acciones que se llevan a cabo (conocimiento que se tiene sobre el tema, y conocimiento que se tiene en cuanto a cómo actuar). De allí se parte que las estrategias metacognitivas están dirigidas a la organización y preparación de lo que se hará.

A propósito de esto, siguiendo a González y Tourón (1992):

Las estrategias metacognitivas
hacen referencia a la planificación,
control y evaluación por parte de
los estudiantes de su propia
cognición. Son un conjunto de
estrategias que permiten el
conocimiento de los procesos
mentales, así como el control y
regulación de los mismos con el
objetivo de lograr determinadas
metas de aprendizaje. (p. 63)

De acuerdo con lo que plantean dichos autores, las estrategias metacognitivas tratan de la organización, inspección y valoración que hace el estudiante a su conocimiento, sobre la base de una serie de maniobras y/o habilidades en virtud de la noción de qué hacer para lograr la adquisición del aprendizaje.

En resumen, las estrategias cognitivas son las empleadas para adquirir el conocimiento, para comprender; mientras que las metacognitivas se emplean en pro de organizar y preparar el conocimiento de lo que se tiene para luego ser aplicado.

Adicionalmente, tomando en cuenta las estrategias cognitivas y su clasificación, en conjunto con las estrategias metacognitivas, vale acotar que Román y Gallegos (1994) establecieron un test basado en considerar que el cerebro funciona (de acuerdo con la representación mental del conocimiento en la memoria) como si fuera la condición de tres procesos cognitivos básicos como los son: a) adquisición, b) codificación o almacenamiento y c) recuperación, ahondado a ello, existe una función de procesamiento natural metacognitivo, el cual se denomina de apoyo.

En relación con lo anteriormente presentado, se destaca el fundamento de cada una según estos autores.

\section{Estrategias de adquisición}

En la adquisición de información se tiene conocimiento que el primer paso para adquirir información es atender, así los procesos atencionales son los encargados de seleccionar y transformar la información desde el ambiente al registro sensorial, esto según el modelo de Atkinson y Shiffrin (1968) que lo visualiza de la siguiente manera:

- Estrategias atencionales: Estas favorecen los procesos atencionales y mediante ellos el control o dirección de todo el sistema cognitivo hacia la información relevante de cada contexto.

- Estrategias de repetición: La repetición tiene como función hacer durar y/o hacer posible y facilitar el paso de la información a la memoria a largo plazo (MLP). Se emplean para repasar de manera constante el material a ser aprendido, se efectúa las acciones más diversas como leer, escuchar, escribir, pensar en el tema (diciéndolo mentalmente) y repetir oralmente. 
- Estrategias de codificación: Aparte de llevarse a cabo los procesos de atención y repetición en el paso de la información de la memoria de corto plazo a la de largo plazo también se activan los procesos de codificación. La elaboración y la organización de la información conectan esta con los conocimientos previos y se integran en lo que se denomina estructura cognitiva o base de conocimiento.

- Estrategias de recuperación: Dado que uno de los factores o variables que explica la conducta de una persona es la información ya procesada, el sistema cognitivo necesita contar con la capacidad de recuperación o recuerdo de ese conocimiento almacenado en la memoria de largo plazo. En tal sentido, estas estrategias son aquellas que favorecen la búsqueda de información en la memoria y la generación de respuesta.

- Estrategias de apoyo al procesamiento: En tanto se realiza el procesamiento de información, otros procesos de naturaleza metacognitiva y no cognitiva, los de apoyo, optimizan, son neutrales o entorpecen el funcionamiento de las estrategias cognitivas de aprendizaje.

Estas estrategias de acuerdo con estos investigadores apoyan, ayudan y potencian el rendimiento de las estrategias de adquisición, de las de codificación y de las de recuperación incrementando así la motivación, la autoestima, la atención, garantizando de esta manera el clima adecuado para un buen funcionamiento de todo el sistema cognitivo.

Enfocados en las acciones pertenecientes a las estrategias cognitivas plasmadas en el test ACRA de Román y Gallegos, garantizando el funcionamiento adecuado del sistema cognitivo; se consideró la factibilidad para que el educando incursione en el proceso de aplicación de estrategias metacognitivas basadas en la implementación de planificación, control y evaluación de las actividades que deberá desarrollar para lograr la comprensión de textos instruccionales para la práctica de juegos deportivos.

Tomando en cuenta lo anteriormente presentado, respecto a las estrategias de aprendizaje y su clasificación, cabe resaltar que uno de los fines del presente trabajo fue identificar cuál de estas o cuáles de estas fueron las aplicadas por los estudiantes al momento de realizar la lectura de textos instruccionales sobre juegos deportivos en Educación Física. Por tanto, a continuación se presenta información sobre dicha disciplina.

Todo lo planteado permite señalar que el objetivo de la presente investigación fue identificar cuáles de las estrategias cognitivas y metacognitivas emplean los estudiantes de Educación Básica para la comprensión de los textos instruccionales utilizados para la práctica de los juegos deportivos en el área de Educación Física.

$\mathrm{Ha}$ de considerarse que según Navarro (s/f), la Educación Física desde la prehistoria, inicia a partir del comienzo del movimiento corporal organizado haciendo alusión a los juegos y bailes de esa época primitiva. A partir de allí, comienza la evolución del origen de la tendencia humana en relación 
con lo establecido en la sociedad, incursionando en esa serie de actividades organizadas ofrecidas solo a los jóvenes varones para que en ellos surgiera el desarrollo de la fortaleza y fuesen más hábiles en términos motores, con el objetivo de realizar actividades de supervivencia como búsqueda continua de alimentos, vestimenta, albergue, protección de un ambiente hostil $y$, la propagación de las especies.

Las transformaciones de esta (Educación Física) fueron evolucionando a través de las diferentes épocas, considerando conceptos referenciales, como son: gimnasia, juegos, deportes, higiene, salud y recreación. De aquí que, a fines del siglo XIX en Argentina, se alcanza su institucionalización como asignatura, $\mathrm{y}$ es en el siglo $\mathrm{XX}$ donde se introduce como profesión.

Vale acotar que en Venezuela comienza a evolucionar a partir de 1936, siendo el Presidente de la República el Gral. Eleazar López Contreras y Ministro de Educación, el Dr. Mariano Picón Salas (este último es quien logra, entre otras metas, crear la Escuela Normal Superior "Instituto Pedagógico Nacional", según decreto 30-09-36). Con el correr de los tiempos se crea la acción de Educación Física, con el fin de capacitar docentes en esta área. Los estudios tenían una duración de tres años. Para dar mayor firmeza al área, se crea la especialidad, en atención al reglamento del Instituto Pedagógico en 1947, para posteriormente en 1962 ser elevado a Departamento por decisión del Consejo Académico del instituto.

Actualmente, a nivel mundial, la Educación Física desde el componente escolarizado, se encuentra dando un auge en relación con la proporción de la globalización de muchos contenidos propios del área y otras asignaturas; así se evidencia, por ejemplo, en España y Argentina.

En el caso de España, están Monzón y García (2012), quienes en esta área promueven la motivación de la lectura, a fin de aportar una alianza en el desarrollo de las competencias lingüísticas y al plan de lectura del Centro Educativo al cual pertenecen (CEIP San Fernando de Maspalomas). Asimismo, se encuentran López (2009) y Morales (2007). Estos promueven, en el nuevo marco legislativo que rige la educación, fomentar la lectura desde todos los departamentos didácticos, incluido el de Educación Física.

En Buenos Aires, Ricci y Sosa (2008) crean un material titulado "La comprensión en Educación Física", destinado a supervisores, equipos de conducción y profesores de Educación Física con el fin de establecer la estrecha vinculación entre la comprensión y la acción, promoviendo la lectura de textos a fin de que los estudiantes construyan su fundamentación acerca de las prácticas corporales.

Ahora bien, en Venezuela, esta área de aprendizaje es considerada un eje integrador, porque "ofrece un desarrollo importante en todas las dimensiones que conforman al ser humano,..." (Currículo de Educación Primaria Bolivariana, 2007, p. 23); además abarca, en su totalidad, al Sistema Educativo Bolivariano (SEB).

Para efectos de esta investigación, se trabajó con los juegos deportivos que, según Piaget (1972)

son aquellas actividades dirigidas y basadas en un código de reglas, 
donde el educando aprende a conocer su cuerpo y a utilizar sus posibilidades de movimiento de forma responsable, ayudándole a adquirir los conocimientos, las destrezas, actitudes y habilidades que le permitan mejorar sus condiciones de calidad de vida y salud (p. 15).

Referente a lo que plantea Piaget (1972), los juegos deportivos representan actividades que bajo reglamento establecido le permite al estudiante indagar sobre su cuerpo, sus posibilidades, movimientos, habilidades y destrezas; de forma tal que pueden adquirir conocimiento que le permite crear conciencia en cuanto a mejoras de sus condiciones de calidad de vida y salud.

En este sentido, se hace referencia a que en la actualidad los juegos deportivos están marcando un gran auge en el ámbito educativo; puesto que está establecido como basamento legal su promoción y participación a través de los llamados "juegos intercursos" y "juegos escolares", ya que habían dejado de ser tomados en cuenta en algunas instituciones.

Ante ello se han venido haciendo llamados a retomar tales actividades y, para hacer énfasis en el tema, se recordó que se encuentra estipulado en la Constitución de la República Bolivariana de Venezuela (1999), en su artículo 111. Allí se establece que "Todas las personas tienen derecho al deporte y la recreación como actividades que beneficien la calidad de vida individual y colectiva... y que se asumirá el deporte y la recreación como política de educación y salud" (s/n).
Valorando lo mencionado en la Constitución (1999) y tomando en cuenta que el Currículo de Educación Primaria Bolivariana (2007) contempla la Educación Física como un área de aprendizaje, se incursionó en lo relativo a la comprensión de la lectura de textos instruccionales usados para la práctica de juegos deportivos, a fin de lograr en los estudiantes la ejecución adecuada de estos y por ende el trabajo autónomo, además de promover, a su vez, el aprendizaje significativo.

Los textos instruccionales, considerando lo manifestado por (Salazar, 2014), representan "el tipo de texto que tiene como función establecer instrucciones, reglas $y$ órdenes... presentan un conjunto de acciones o pasos presentados en el mismo orden en que deben realizarse." (p. 2). Tienen como función establecer instrucciones, reglas y órdenes. Estos hacen énfasis en la ejecución de una serie de acciones que se encuentran plasmadas en el orden en que deben ser llevadas a cabo.

Es evidente que para el desarrollo de las acciones que se encuentran plasmadas en los textos instruccionales, es de vital importancia llevar a cabo un proceso lector que implique la comprensión de lo establecido en cada párrafo a ejecutar.

Para Rincón (2014)

La lectura es el proceso de la recuperación y la aprehensión de algún tipo de información o ideas almacenadas en un soporte $y$ transmitidas mediante algún tipo de código, usualmente el lenguaje, que puede ser visual o táctil como por ejemplo el sistema Braille ( $s / n)$. 
En este sentido, según este autor, la lectura implica, a través de una serie de elementos, la adquisición de la información y su codificación para luego ser transmitida de forma oral o escrita.

Por otra parte, Ibarra (s.f.) dice que

es importante indicar que durante décadas la idea básica sobre la lectura ha sido que leer es poco más que reconocer ciertos símbolos gráficos y asociarlos a sonidos para transformar lo escrito en oral y aplicar luego, sobre ese discurso ya oralizado, las habilidades de comprensión de la lengua hablada (p. 1).

Respecto a esto, la aplicación de habilidades lectoras permite transformar lo leído cuando existe comprensión. Esto se da por medio de la asociación de símbolos y sonidos que suelen ser en forma oral. Así "... aprender a leer no era sino adquirir la capacidad de reconocer tales símbolos y aprender las reglas que los asocian a los sonidos" (Ibarra, s.f., p. 1).

De esta manera, se parte que la lectura no solo represente una acción de aprehensión de información, sino también que ella debe tener como fin la comprensión de lo que se establece en la información a través de la capacidad de identificar símbolos y reglas que se asocien a sonidos, palabras o hechos y vivencias.

En cuanto a la comprensión de la lectura, entre las décadas de los años 60 y 70, Cooper y Coller (1990) consideraron que era el resultado directo de la decodificación o conversión de los grafemas escritos a los sonidos.
En el transcurrir de esas décadas, la idea que imperaba era que si los estudiantes eran capaces de denominar las palabras, la comprensión tendría lugar de forma automática. Poco a poco los mismos profesores fueron comprobando que muchos estudiantes seguían sin comprender los textos escritos y que la comprensión no tenía lugar de forma automática, a pesar de que el interés se hubiese centrado en la decodificación. Es decir, no solo bastaba con el hecho de que los estudiantes tuvieran dominio de las palabras para que a través de la codificación de estas se diera lugar a la comprensión de la lectura.

Torres (2014) mostró, por primera vez, en el Laboratorio Latinoamericano de Evaluación de Calidad en la Educación (LLECE) de la UNESCO, un modelo que cotejaba el logro educativo en países que comparten una cultura con rasgos esenciales comunes. El estudio fue empleado a estudiantes del tercer y cuarto grado de Educación Primaria.

Los resultados derivados de este modelo en relación con el lenguaje fueron fraccionados en tres (3) grupos: un primer grupo que correspondió al promedio esperado (400), donde Cuba dominó el primer lugar; un segundo grupo cuyo promedio se ubicó por arriba de la media regional de lo esperado (250 en adelante), este grupo estuvo constituido por Argentina, Brasil, Colombia, Chile, México, Bolivia y Paraguay, y por último, un tercer grupo formado por los países que quedaron por debajo del promedio (250), entre ellos Costa Rica, Honduras, República Dominicana, Perú y Venezuela (que ocupó el penúltimo lugar de nivel más bajo). 
Este asunto se volvió de gran importancia, ya que durante la década de los años 90, la educación se transformó en la principal política pública en América Latina.

El primer estudio de comparación del logro educativo aplicada por la UNESCO, fue denominada PERCE (Primer Estudio Regional Comparativo y Explicativo, 1997). Cabe resaltar que luego de ello, en el año 2000, fue aplicado un segundo estudio llamado SERCE (Segundo Estudio Regional Comparativo y Explicativo), y seguidamente en 2013 un tercer estudio que llevó por nombre TERCE (Tercer Estudio Regional Comparativo y Explicativo), tomando en cuenta que Venezuela no participó en los dos últimos.

Desde el año 1998 Venezuela no ha realizado estudios de evaluación a sus estudiantes a nivel nacional, en relación con el promedio de avance educativo en sus principales áreas de aprendizaje: Lengua y Matemática. Sin embargo, a partir de 1999 hasta el 2014, según Consulta Nacional por la Calidad Educativa (CENAL, 2014), se ha venido trabajando en pro de mejorar la calidad educativa, a partir de: a) la incorporación de los ejes: trabajo liberador, desarrollo endógeno sustentable, desarrollo de valores éticos y espirituales, así como también gestión comunitaria, entre otros; b) la inclusión de las áreas de aprendizaje: Antropología Histórica y Arqueología, Prevención y Gestión de Riesgo, Lenguas e Idiomas Autóctonas, y otras más; c) la participación activa de miembros de la comunidad, todo esto suscrito en el Proyecto Educativo Integral Comunitario (PEIC); e) el apoyo de las misiones con fines educativos: Ribas y Robinson; f) la donación de libros de textos y g) el sistema educativo de enseñanza tecnológica con las canaimitas.

Sin embargo, se debe considerar el informe del año 2012 del estado bolivariano de Miranda, titulado "El significado del Cambio en Educación". Allí se señala que por iniciativa del gobierno regional a partir de 2009 se realizaron asambleas en las que asistieron más de 1.500 docentes y directivos, con el propósito de plantear propuestas para mejorar la calidad educativa.

Luego, a partir de 2011 se crearon planes que permitieron mejorar la calidad educativa en cuanto al proceso de enseñanza y aprendizaje de los estudiantes del estado Miranda, por eso surge, entre otros, el Plan Integral de Lectura y Alfabetización Social (PILAS, 2009), que proponía algo sencillo pero muy poderoso, como que todos los días en todas las escuelas todos los niños lean y escriban hasta que lo logren correctamente.

La evaluación de estos planes se realiza anualmente en todas las escuelas dependientes de este estado, aplicando pruebas de rendimiento estudiantil a estudiantes de $3^{\text {er }}$ y $6^{\text {to }}$ grado del Subsistema de Educación Primaria y a estudiantes de $3^{\text {er }}$ año de Educación Media General. Estas pruebas incluyen cálculos, resolución de problemas, y análisis de casos matemáticos, así como también lectura de palabras por minutos y comprensión de la lectura.

De acuerdo con lo señalado, se observaron los resultados obtenidos entre el año 2009 y 2012, lo que evidenció un avance sostenido en el área de Lengua. Se ha de acotar que en 2009 con la aplicación de las pruebas de rendimiento aplicadas anualmente, el ente gubernamental decide incorporarse a la evaluación internacional 
PISA (Programa para la Evaluación Internacional de Alumnos) que realiza la Organización para la Cooperación y el Desarrollo Económico, de allí, el estado obtuvo un promedio similar al de México y Uruguay, por debajo de los promedios mundiales, y muy distante de los países con mejor desempeño.

Expuesto lo anterior, cabe resaltar que, en la actualidad, diversos diagnósticos como el de "Primer Encuentro de Directores y Maestros año escolar 2015-2016", realizado en la sede de la Dirección de Educación de la gobernación del estado Miranda en el año 2015 en relación con la aplicación de las pruebas de rendimiento en el estado bolivariano de Miranda, han determinado el bajo promedio que se han venido presentando desde 2012, tomando en cuenta que en el plan PILAS, los docentes le están dando mayor importancia a la cantidad de palabras leídas por minutos, que al texto o tema que presenta el autor en relación a la propia comprensión de la lectura.

Ha de considerarse que Lerner (2001), en su libro Leer y escribir en la escuela: lo real, lo posible y lo necesario, específicamente, en relación con lo necesario, plantea crear estrategias que vinculadas con el quehacer de los estudiantes, permitan despertar el interés por la lectura en cuanto a cuentos, novelas, historia de diversos países, entre otros textos que les sean de gran agrado, promoviendo así el hábito de lectura, así como también al plantear la vinculación de sus quehaceres, resaltar la interacción que este tiene con su ambiente físico y social.

Es en ese contexto donde se visualiza la problemática, ya que las debilidades que presentan los estudiantes en el nivel primario de la educación en Miranda y similar en el territorio nacional venezolano, no son muy alentadoras y esto puede afectar el futuro de la sociedad venezolana, en función de formar un ciudadano consciente, crítico, capaz y conocedor del mundo y sus relaciones, lo que significa un atraso en todos los ámbitos. Y el área de Educación Física no escapa de ello, ya que uno de sus fines, según el Currículo de Educación Primaria Bolivariano (2007) es que el individuo pueda "...crearse en un primer momento una representación en el plano mental de las acciones motrices que debe ejecutar, para luego, mediante un proceso de análisis, manifestar a través de respuestas motoras el resultado de dicho proceso reflexivo" (p. 23). Además, es un componente educativo que está orientado al trabajo del ser humano en su totalidad, debido a que a través de ella se busca no solo el desarrollo de destrezas motoras, habilidades deportivas e integrales, sino también la de índole cognitivo, de valores, conocimiento de su propio ser, tomando también en cuenta el desarrollo intelectual del mismo.

Los juegos son vistos como una serie de actividades recreativas que implican el empleo del tiempo libre, mediante las cuales se toman en consideración reglas a seguir para un mayor disfrute.

En el programa de Educación Física de la Primera Etapa de Educación Básica (1997) se indica que el juego es:

una forma de aprendizaje natural en el alumno; no solo de conocimientos motores, sino también es un medio de aprendizaje social, cognitivo intelectual y experiencial. Es un medio peculiar 
de enfrentarse a las diversas situaciones y que no le ocasione un conflicto, sino una diversión. (p. 85)

En este aspecto, se tiene que los juegos conforman un medio de aprendizaje basado en actividades recreativas que contemplan reglas a seguir para su disfrute.

Por otra parte, los deportes representan actividades físicas que también poseen reglas, pero que, a diferencia de los juegos, tienen un enfoque competitivo.

De igual manera, Zambrano y López (1993) establecen que los deportes "son actividades predominantemente físicas y formativas, las cuales traen como consecuencia el ajuste de la conducta, la personalidad, la agresividad, compañerismo, además de los beneficios anatómicofisiológicos, compitiendo con caballerosidad, lealtad y honestidad" (p. 12).

Sobre la base de los autores anteriormente mencionados, cabe resaltar que los deportes son actividades físicas de formación que tienden a mejorar la conducta, disminuyendo la ofuscación y fomentando la competitividad de forma leal y honesta.

A propósito de los deportes, cabe resaltar que Parlebas (1981) divide los deportes en dos grandes grupos:

- Los psicomotrices: en los que el sujeto participa de forma individual y la presencia de otros sujetos no es esencial (salto de longitud, escalada, gimnasia artística).

Este tipo de deporte también es denominado individual, ya que el deportista, atleta $\mathrm{o}$ aquel que lo practique compite sobre la base de sí mismo. Entre ellos se encuentran el atletismo y la natación.

El otro grupo que plantea Parlebas (1981) se refiere a:

- Los sociomotores: en los que participan varios sujetos que inciden de forma directa en las conductas que desarrollan sus compañeros y/o adversarios.

A estos deportes se les suele llamar colectivos, ya que en ellos se incluye el trabajo en conjunto y cooperativo entre dos o más personas, tomando en cuenta que la acción competitiva es dada por el enfrentamiento entre dos grupos de personas (denominados equipos). En ellos podemos mencionar el baloncesto, la gimnasia rítmica, voleibol, futbolito, carrera de relevos, ciclismo en rutas por equipos, entre otros.

Sobre la base de los términos anteriormente desarrollados (juegos $y$ deportes), se puede definir que los juegos deportivos son aquellos que fusionan distintas facetas de juegos y deportes, basados en el desarrollo motriz de las cualidades físicas, tomando en cuenta una serie de reglas que crean un ambiente competitivo donde se propicia un estímulo mental con base en un aprendizaje social y cognitivo intelectual.

De allí que sea necesario considerar la teoría del juego, según Fernández (2005), la cual

estudia de manera formal y abstracta las decisiones óptimas que deben tomar diversos adversarios en conflicto, pudiendo definirse como el estudio de 
modelos matemáticos que describen el conflicto y la cooperación entre entes inteligentes que toman decisiones. Tales decisiones se consideran estratégicas, es decir, que los entes que participan en el juego actúan teniendo en cuenta las acciones que tomarían los demás. (p. 1)

En cuanto a la Educación Física, el currículo del subsistema de Educación Primaria Bolivariana (2007), que es el contexto de la presente investigación, plantea que

El desarrollo de la actividad física, el deporte y la recreación posee un carácter consciente que permite a los niños y las niñas crearse en un primer momento una representación en el plano mental de las acciones motrices que debe ejecutar, para luego, mediante un proceso de análisis, manifestar a través de respuestas motoras el resultado de dicho proceso reflexivo. (p. 23)

En efecto, aquí se hace alusión que a través de la Educación Física se desarrollan actividades físicas, de deportes y de recreación (inclusión de juegos y actividades de esparcimiento didáctico), mediante las cuales se busca crear en los estudiantes no solo el despliegue de acciones motrices, sino también el desarrollo cognitivo de sus propias potencialidades y destrezas, tomando en cuenta el poder dar respuesta a un estado mental reflexivo sobre sí mismo, una manera de lograrlo es a partir del uso de textos escritos, los cuales son utilizados cuando hay que documentarse, en este caso, sobre los juegos deportivos.

\section{MÉTODO}

El presente estudio estuvo enmarcado dentro del paradigma cuantitativo porque se buscó confirmar el uso de las estrategias de aprendizaje cognitivas y metacognitivas, y a su vez la significatividad que estas tienen en relación con la comprensión de la lectura, tomando en cuenta la frecuencia con la que son empleadas durante el proceso lector de textos instruccionales para lograr la práctica de juegos deportivos. Además, se abordó el diseño de campo descriptivo. Según Arias (1997a , 2997b) "consiste en la caracterización de un hecho, fenómeno, individuo o grupo, con el fin de establecer su estructura o comportamiento" (p. 24).

La población estuvo constituida por los estudiantes de Educación Básica de la U.E.E.B "Ricardo Zuloaga", del año escolar 20162017. Todos estos participantes representaron el $100 \%$ de la población, los cuales residen en sectores aledaños a su centro educativo.

La muestra fue de origen intencional, según Sabino (1992) se "seleccionan los elementos con base en criterios o juicios del investigador" (p. 53). Estuvo basada en un estudio desde que cursaban el $3^{\text {er }}$ grado y fueron observados desde el momento en que se les asignó como actividad el trabajo de lectura de textos instruccionales para la práctica de juegos deportivos, ya que se evidenció en ellos dificultad para su ejecución. Por tanto, la muestra estuvo conformada por los 19 alumnos de 5to. Grado de la institución ya mencionada. 
La técnica fue la observación. Los instrumentos para la observación fueron, en primer lugar, el test ACRA, creado por Román y Gallegos (1994). Este representa una escala que tiene por objeto identificar las estrategias cognitivas y metacognitivas más frecuentemente utilizadas por los estudiantes cuando están asimilando la información contenida en un texto. Se escogió este instrumento porque permitió representar los diferentes tipos de estrategias de aprendizaje que fueron seleccionadas.

Por otra parte, aunque fue diseñado para estudiantes españoles de Educación Secundaria obligatoria, las pruebas de validez y confiabilidad, descritas más adelante, determinaron que podía utilizarse para la Educación Primaria de nuestro país. Estuvo integrado por dieciocho ítems en relación con acciones que llevan a cabo los estudiantes al realizar la lectura de los textos instruccionales en sus fases para la práctica de juegos deportivos; en dicho instrumento se hace mención a los distintos tipos de estrategias cognitivas y metacognitivas, según Román y Gallegos (1994): adquisición de la información, codificación de información, recuperación de información y de apoyo de procesamiento, que se consideraron en cada momento de la lectura (antes, durante y después).

El procedimiento desarrollado en la presente investigación, se dividió en dos etapas (cada una de ellas estuvieron constituidas por distintas fases):

\section{Etapa I: Diagnóstico}

$\mathbf{1}^{\text {ra }}$ fase: Evaluación por Juicio de expertos para la validación del Test ACRA $\mathbf{2}^{\text {da }}$ fase: Aplicación del Test ACRA

\section{Etapa II: Análisis e interpretación de los resultados}

$\mathbf{1}^{\text {ra }}$ fase: Análisis de los resultados

$2^{\text {da }}$ fase: Interpretación de los resultados

RESULTADOS

Para el análisis de los resultados, se tomó en cuenta la cantidad de respuestas, según cada pregunta del test ACRA y cada uno de los estudiantes que dieron respuesta. Seguidamente se desglosó cada ítem, por fase de la lectura (antes, durante y después), con la finalidad de realizar la descripción de los resultados, en virtud de establecer con ello el total de cantidad de respuestas, según las frecuencias de uso y posteriormente determinar las estrategias que más emplearon, y así presentar el análisis de los resultados.

A continuación, se procede a explicar los resultados arrojados en cada ítem.

\section{Antes de la lectura}

En la fase antes de la lectura, se evidenció que al inicio de la lectura predominaron tres acciones correspondientes a las estrategias de adquisición de información, dos de las estrategias de codificación y una de las estrategias de apoyo al procesamiento.

En cuanto a la opción que más indicaron al seleccionar las acciones de los tipos de estrategias, se obtuvo que fue "algunas veces", la cual representó el 63,15\%. Esto permitió determinar que la estrategia "adquisición de información" fue la más empleada, debido a que antes de iniciar la 
lectura de un texto instruccional para la práctica de juegos deportivos, lo primero que los estudiantes leen es el título y el resumen y/o introducción.

\section{Durante la lectura}

En la fase "durante la lectura, se determinó, que la estrategia que emplean "siempre", con un 52,63\%, es la decodificación, haciendo mención a la acción de buscar ayuda con el profesor o los compañeros cuando tienen alguna duda sobre alguna instrucción que desconozcan del texto; y "algunas veces", con un 52,63\%, es la estrategia de "recuperación", cuando al leer un texto instruccional para la práctica de juegos deportivos, utilizan la imaginación para ver lo que están leyendo como si fuera una película y así poder ejecutarlo.

\section{Después de la lectura}

La fase "después de la lectura", se estructuró en relación con cuatro acciones: una de adquisición de información, dos de recuperación de información y una de apoyo al procesamiento.

En lo que a esto concierne, un 52,63\% determinó que, "algunas veces", ejecutan una de las acciones de las estrategias de recuperación de la información, la cual se refiere a que antes de plantear la interpretación del contenido, van recordando palabras, imágenes o dibujos relacionados con las ideas principales del texto instruccional que leyeron para la ejecución práctica de juegos deportivos.

Luego de haber explicado los resultados arrojados en cada uno de los ítems y en relación con las acciones de las estrategias empleadas en el proceso lector (antes, durante y después de la lectura), se presenta la Tabla 1, en el que se da a conocer el total de respuestas por cada una de la frecuencia de ejecución.

Tabla 1. Total de cantidades de respuestas según la frecuencia

\begin{tabular}{lcc}
\hline \multicolumn{1}{c}{ Frecuencia de ejecución } & Cantidad de respuestas & Porcentaje \\
\hline Nunca & 55 & 16,08 \\
Algunas veces & 117 & 34,21 \\
Casi siempre & 85 & 24,85 \\
Siempre & 85 & 24,85 \\
Total de respuestas a las frecuencias & 342 & 100 \\
\hline
\end{tabular}

Allí se puede apreciar que el total de respuestas dadas por los estudiantes es de 342 , lo que representa el $100 \%$; de este total 55 respuestas reflejan el $16,08 \%$ de la frecuencia "nunca"; 117 respuestas personifican el $34,21 \%$ de la frecuencia "algunas veces"; 85 de ellas muestran que el $24,85 \%$ son de la frecuencia "casi siempre", y finalmente otras 85 respuestas representan el $24,85 \%$ de la frecuencia "siempre". Todo esto apunta hacia el empleo de estrategias cognitivas y metacognitivas para lograr la comprensión de la lectura de textos instruccionales para la práctica de juegos deportivos en Educación Física. 
En la Tabla 2, se muestra la distribución de cada frecuencia según las estrategias cognitivas y metacognitivas empleadas. Allí se evidencia que la estrategia de "codificación de información" fue la que más emplearon los estudiantes con 33 cantidades de respuesta en frecuencia de "casi siempre".

Tabla 2. Estrategias que emplean los estudiantes

\begin{tabular}{lcccc}
\hline \multicolumn{1}{c}{ ESTRATEGIAS } & \multicolumn{4}{c}{ FRECUENCIA } \\
& Nunca & Algunas veces & Casi siempre & Siempre \\
\hline Adquisición de información & 11 & 34 & 21 & 29 \\
Codificación de información & 24 & 27 & 33 & 30 \\
Recuperación de información & 9 & 28 & 11 & 9 \\
Apoyo al procesamiento & 11 & 28 & 20 & 17 \\
\hline
\end{tabular}

Finalmente, luego de verificar la frecuencia del empleo de estrategias cognitivas y metacognitivas por parte de los estudiantes para la comprensión de textos instruccionales de los juegos deportivos de Educación Física, y considerando la cantidad de respuestas dadas a cada ítem (del 1 al 18), se observó que del total de respuestas, el mayor número estuvo representado en la frecuencia de uso "algunas veces", la cual arrojó un porcentaje de $34,21 \%$. Esto permitió evidenciar lo expuesto en el planteamiento del problema, en el que se señalaba la necesidad de promocionar el uso de estas estrategias para mejorar la comprensión de la lectura en los tipos textos ya mencionados, con el fin de lograr que los estudiantes logren ejecutar con una mejor eficiencia los juegos deportivos: individuales y colectivos.

Es sabido que las estrategias de aprendizaje (cognitivas y metacognitivas) les proporcionan a los estudiantes la capacidad de adquirir y desarrollar su propio aprendizaje de forma significativa. Sin embargo, los datos arrojados en la primera fase diagnóstica de la investigación muestran que los estudiantes las ponen en práctica "algunas veces", y que la más utilizada es la del tipo "codificación de información".

Ante ello, es importante recordar que, según Román y Gallego (1994), el cerebro funciona de acuerdo con la representación mental del conocimiento en la memoria como si fuera la condición de tres procesos cognitivos básicos, como los son: a) adquisición, b) codificación o almacenamiento y c) recuperación, ahondado a ello, existe una función de procesamiento natural metacognitivo, el cual se denomina de apoyo. Es decir, que para la obtención de resultados significativos respecto al uso de estrategias cognitivas y metacognitivas, lo ideal es la aplicación de los cuatro procesos representados en los distintos tipos de estrategias: a) Estrategias de adquisición, b) estrategias de codificación, c) estrategias de recuperación, y d) estrategias de apoyo de procesamiento.

Esto lleva a la valoración final de que por la poca frecuencia con la cual los estudiantes emplean estrategias cognitivas $y$ metacognitivas, y por el mayor uso de solo una de ellas (estrategias de codificación de 
información), se pudo determinar que es de vital importancia ahondar en la solución del problema planteado en la presente investigación, por medio de la promoción de estrategias cognitivas y metacognitivas que tengan como finalidad incentivar, por parte de los estudiantes, la aplicación de dichas estrategias para la comprensión de la lectura de textos instruccionales utilizados para la práctica de juegos deportivos.

\section{CONCLUSIONES}

Sobre la base del objetivo planteado y los resultados arrojados en la presente investigación, vale resaltar que para dar respuesta a cuáles estrategias cognitivas y metacognitivas empleaban los estudiantes para lograr la comprensión de textos instruccionales para la práctica de juegos deportivos, se procedió a considerar una fase diagnóstica con la aplicación del Test ACRA de Román y Gallegos (1994). Dicho instrumento arrojó como resultado que las estrategias que más empleaban era la de "codificación de información".

Según estos resultados, se determinó lo establecido por Román y Gallegos (1994), en relación con que el cerebro funciona con sus tres procesos cognitivos (información codificación y recuperación), tomando en cuenta un cuarto procedimiento, natural metacognitivo que denomina de apoyo. Para ello, se enfatizó en la necesidad de dar a conocer a los estudiantes (sujetos de estudio) lo importante que es emplear los tres procesos cognitivos y el metacognitivo natural, todos enfocados en las estrategias ya mencionadas, las cuales a su vez implican acciones que ellos llevan a cabo durante el proceso lector, aunque no sean conscientes de que las emplean.

En fin, queda demostrado, una vez más, que el uso de las estrategias cognitivas y metacognitivas permite dar respuestas positivas y resultados significativos, en este caso en particular, durante el proceso de la lectura de textos instruccionales para la práctica de juegos deportivos en el área de Educación Física.

\section{REFERENCIAS}

Arias, F. (1997a). El proyecto de la investigación. Guía para su elaboración. Caracas: Episteme

Arias, F. (1997b). El proyecto de la Investigación Científica. Introducción a la metodología científica. Caracas: Editorial Episteme

Atkinson, R. y Shiffrin, R. (1968). La memoria humana: A sus procesos de control de sistema y propuesta. En K. W. Spence (Ed.). La psicología del aprendizaje y la motivación: avances en la investigación y la teoría, vol. 2. Nueva York: Academic Press

CENAL (2014). I Consulta Nacional por la Calidad educativa. Recuperado de https://xa.yimg.com/kq/groups/9509988 /438168472/name/1+Consulta+Nacional + por+la+Calidad+Educativa +en ++BOX. pdf

Cooper, J. y Coller, J. (1990). Cómo mejorar la comprensión lectora. Madrid: Visor

Constitución Nacional de la República Bolivariana de Venezuela (1999). Gaceta Oficial de la República Bolivariana De Venezuela No 5.453 Extraordinario. Caracas 24 de Marzo

Currículo del Subsistema de Educación Primaria Bolivariana. Caracas, septiembre de 2007

Fernández, F. (2005). Teoría de juegos: análisis matemático de conflictos. Curso 
Interuniversitario "Sociedad, Ciencia, Tecnología y Matemáticas

Gobierno de Miranda. Educación. Dirección de Educación (2015). 1er. Encuentro de Directores y Maestros. Año escolar: 2015-2016. Recuperado der http://www.miranda.gob.ve/educacion/i mages/archivos_pdf/direcmaes.pdf

González, M. y Tourón, J. (1992). Autoconcepto y rendimiento académico. Sus implicaciones en la motivación y en la autorregulación del aprendizaje. Pamplona: EUNSA

Ibarra, S. (s/f). Los Procesos de Lectura Bases Teóricas. Recuperado de http://www.academia.edu/15208898/LOS _PROCESOS_DE_LECTURA_BASES_TE\%C3 $\% 93 R I C A S$

Lerner, D. (2001). Leer y Escribir en la Escuela: lo real, lo posible y lo necesario. México: Editorial: FCE

López, J. (2009). Cómo afrontar el fomento de la lectura desde la Educación Física. Propuestas prácticas. España. Recuperado der http://www.efdeportes.com/efd137/elfomento-de-la-lectura-desde-laeducacion-fisica.htm

Monzón, J. y García, G. (2012). ¿La biblioteca en el gimnasio?: aportación desde el área de Educación Física al desarrollo del plan de lectura y de la competencia lingüística, Canarias. Recuperado de http://www3.gobiernodecanarias.org/me dusa/ecoescuela/tamadaba/2012/01/11/l a-biblioteca-en-el-gimnasio-aportaciondesde-el-area-de-educacion-fisica-aldesarrollo-del-plan-de-lectura-y-de-lacompetencia-linguistica/

Morales, R. (2007). Fomento de la Lectura a través del área de Educación Física en Primaria. Melilla, España: IES Enrique Nieto

Navarro, J. (s/f). Origen y evolución de la Educación Física el Deporte y la
Recreación en Venezuela. UNIDAD III. [Documento impreso]

Parlebas, P. (1981). Contribución a un lexique commenté en science de l'action motrice. París: INSEP

PERCE (1997). Primer Estudio Internacional Comparativo sobre Lenguaje, Matemática y Factores Asociados en Tercero y Cuarto Grado de Educación Básica. Recuperado de http://www.unesco.org/new/es/santiago/ education/education-assessmentllece/perce-serce-databases/

Piaget, J. (1972). Los estadios del desarrollo del niño y del adolescente. Barcelona, España: Editorial Sen

PILAS (2009). Plan Integral de Lectura y Alfabetización Social. Gobierno de Miranda

Ricci, A. y Sosa, R. (2008). La comprensión en Educación Física. Recuperado de http://servicios.abc.gov.ar/lainstitucion/si stemaeducativo/educacionfisica/docume ntos/documentosdescarga/capacitacion/ la comprension en educacion fisica.pdf

Rincón, M (2014). Historia de la Lectura. Recuperado de Mayraalejandra2.wordpress.com

Román, J. y Gallegos, S. (1994). Escala de Estrategias de Aprendizaje. Publicaciones de Psicología Aplicada. Madrid, España: TEA Ediciones. S.A

Sabino, C. (1992). El Proceso de la Investigación. Caracas: Editorial PANAPO

Salazar, I. (2014). Habilidad verbal. El texto instruccional. [Material audiovisual en línea]. Recuperado de http://es.slideshare.net/ingrids12/textoinstruccional

SERCE (s/f). Los Aprendizajes de los Estudiantes de América Latina y el Caribe. Recuperado de unesdoc.unesco.org/images/0016/00160 6/160660s.pdf [Consulta: domingo 26, abril 2015] 
Torres, R. (2014). América Latina y las Pruebas LLECE. Recuperado de http://otraeducación.blogsport.com/2014/12/ameri ca-latina-y-las-pruebas-llece.htm

Weihrich, H. y Koontz, H. (1993). Administración, una perspectiva global. México: Ed. McGraw Hill
Zambrano, C. y López, L. (1993). Nuestra Educación Física de hoy. $7^{0}$ grado. Caracas: Ediciones CO-BO 